\title{
Eyelid Pressure and Contact with the Ocular Surface
}

\author{
Alyra J. Shaw, Michael J. Collins, Brett A. Davis, and Leo G. Carney
}

Purpose. To investigate static upper eyelid pressure and contact with the ocular surface in a group of young adult subjects.

Methods. Static upper eyelid pressure was measured for 11 subjects using a piezoresistive pressure sensor attached to a rigid contact lens. Measures of eyelid pressure were derived from an active pressure cell (1.14-mm square) beneath the central upper eyelid margin. To investigate the contact region between the upper eyelid and the ocular surface, the authors used pressuresensitive paper and the lissamine-green staining of Marx's line. These measures, combined with the pressure sensor readings, were used to derive estimates of eyelid pressure.

Results. The mean contact width between the eyelids and the ocular surface estimated using pressure-sensitive paper was $0.60 \pm 0.16 \mathrm{~mm}$, whereas the mean width of Marx's line was $0.09 \pm 0.02 \mathrm{~mm}$. The mean central upper eyelid pressure was calculated to be $3.8 \pm 0.7 \mathrm{~mm} \mathrm{Hg}$ (assuming that the whole pressure cell was loaded), $8.0 \pm 3.4 \mathrm{~mm} \mathrm{Hg}$ (derived using the pressure-sensitive paper imprint widths), and $55 \pm 26 \mathrm{~mm} \mathrm{Hg}$ (based on contact widths equivalent to Marx's line).

Conclusions. The pressure-sensitive paper measurements suggested that a band of the eyelid margin, significantly larger than the anatomic zone of the eyelid margin known as Marx's line, had primary contact with the ocular surface. Using these measurements as the contact between the eyelid margin and the ocular surface, the authors believe that the mean pressure of $8.0 \pm 3.4$ $\mathrm{mm} \mathrm{Hg}$ is the most reliable estimate of static upper eyelid pressure. (Invest Ophthalmol Vis Sci. 2010;51:1911-1917) DOI: 10.1167/iovs.09-4090

$\mathrm{T}$ he eyelid is in close contact with the ocular surface, but the exact pressure and area of contact between the two surfaces is unknown. Previous investigations suggest that the eyelid margin is the main region of the eyelid in contact with the ocular surface. X-ray examination of an human upper eyelid showed close contact between the eyelid margin and the cornea. ${ }^{1}$ This area of the marginal conjunctiva has been termed the lid wiper because it is thought to be involved in the distribution of the tear film layer during blinking. ${ }^{2,3}$ Increased staining in this region in dry eye subjects (lid-wiper epitheliopathy) also suggests enhanced frictional contact of this region with the ocular surface. ${ }^{2-5}$ Another anatomic feature of the eyelid margin is Marx's line. ${ }^{6}$ This narrow line of squamous cells extends along the entire length of the upper and lower eyelids and can be visualized by staining with rose Bengal or

From the Contact Lens and Visual Optics Laboratory, School of Optometry, Queensland University of Technology, Brisbane, Queensland, Australia.

Submitted for publication June 4, 2009; revised September 15, 2009; accepted September 21, 2009.

Disclosure: A.J. Shaw, None; M.J. Collins, None; B.A. Davis, None; L.G. Carney, None

Corresponding author: Alyra J. Shaw, Contact Lens and Visual Optics Laboratory, School of Optometry, Queensland University of Technology, Victoria Park Road, Kelvin Grove, Queensland, Australia, 4059; aj.shaw@qut.edu.au. lissamine green vital dyes. ${ }^{6-8}$ The squamous cell phenotype of this tissue and the staining properties of this region suggest that it is subject to mechanical contact and may be involved in contacting the ocular surface..$^{7,9,10}$

Three studies have been published in the past five decades in which the authors designed systems to attempt to measure eyelid pressure (Shikura H, et al. IOVS 1993;34:ARVO Abstract 1250). ${ }^{11,12}$ These techniques used modified contact lenses to create a chamber that was filled with either air or water and that was attached to a manometer. The applied pressure was measured from the change in level of the manometer fluid column. These devices were used to measure eyelid pressure, whereas the subjects were instructed to perform gentle or forced blinks, and static eyelid pressure was not reported. The contact lens devices were relatively thick (up to $2.5 \mathrm{~mm}$ ), ${ }^{12}$ which might have caused distension of the eyelids and influenced the accuracy of the measurements.

The aim of this study was to quantify static upper eyelid pressure (without blinking) using a thin $(0.17-\mathrm{mm})$ pressure sensor mounted on a contact lens ${ }^{13}$ for a group of young adult subjects. Because of the dimensions of the pressure measuring cells within the pressure sensor, the contact region between the upper eyelid and the ocular surface had to be considered. As the contact region cannot be directly visualized, three models of eyelid contact with the pressure cell were based on the sensor's pressure cell dimensions, images of Marx's line, and eyelid margin contact imprints derived from pressure-sensitive paper. These were used to scale the pressure data to estimate the pressure of the upper eyelid margin on the ocular surface.

\section{Subjects ANd Methods}

Eleven subjects were recruited from the staff and students of the Queensland University of Technology School of Optometry. There were seven female and four male subjects of Caucasian $(n=6)$, Indian $(n=4)$, and Iranian $(n=1)$ ethnicity with a mean age of $28 \pm 3$ years (range, 22-33 years). Subjects were close to emmetropic with a bestcorrected acuity of $0.00 \log$ MAR or better, a mean spherical refractive error of $+0.10 \pm 0.39 \mathrm{D}$, and a mean astigmatism of $-0.20 \pm 0.31 \mathrm{D}$. This research was approved by the university research ethics committee and adhered to the tenets of the Declaration of Helsinki. Written consent was obtained from each study participant.

To confirm that all the inclusion and exclusion criteria were satisfied, all subjects underwent preliminary eye examinations. Slit-lamp biomicroscopy confirmed a clear and healthy cornea with no evidence of dry eye, Meibomian gland dysfunction, blepharitis, entropion, ectropion, chalazia, or ptosis. Dry eye was defined as a score of $\geq 14$ on the McMonnies dry eye symptom survey and a noninvasive tear break-up time of $<10$ seconds using projected mires. ${ }^{14}$ Subjective refraction, using the maximum plus for best visual acuity criteria, was used to confirm refractive status. As there is evidence that rigid contact lens wear can lead to slight ptosis ${ }^{15-18}$ and there are anecdotal reports of ptosis from soft contact lenses, ${ }^{19}$ all contact lens wearers were excluded from the study. Subjects also had no history of ocular surgery or injury. Due to the eyelid pressure apparatus set-up, the right eye was investigated for each subject. 

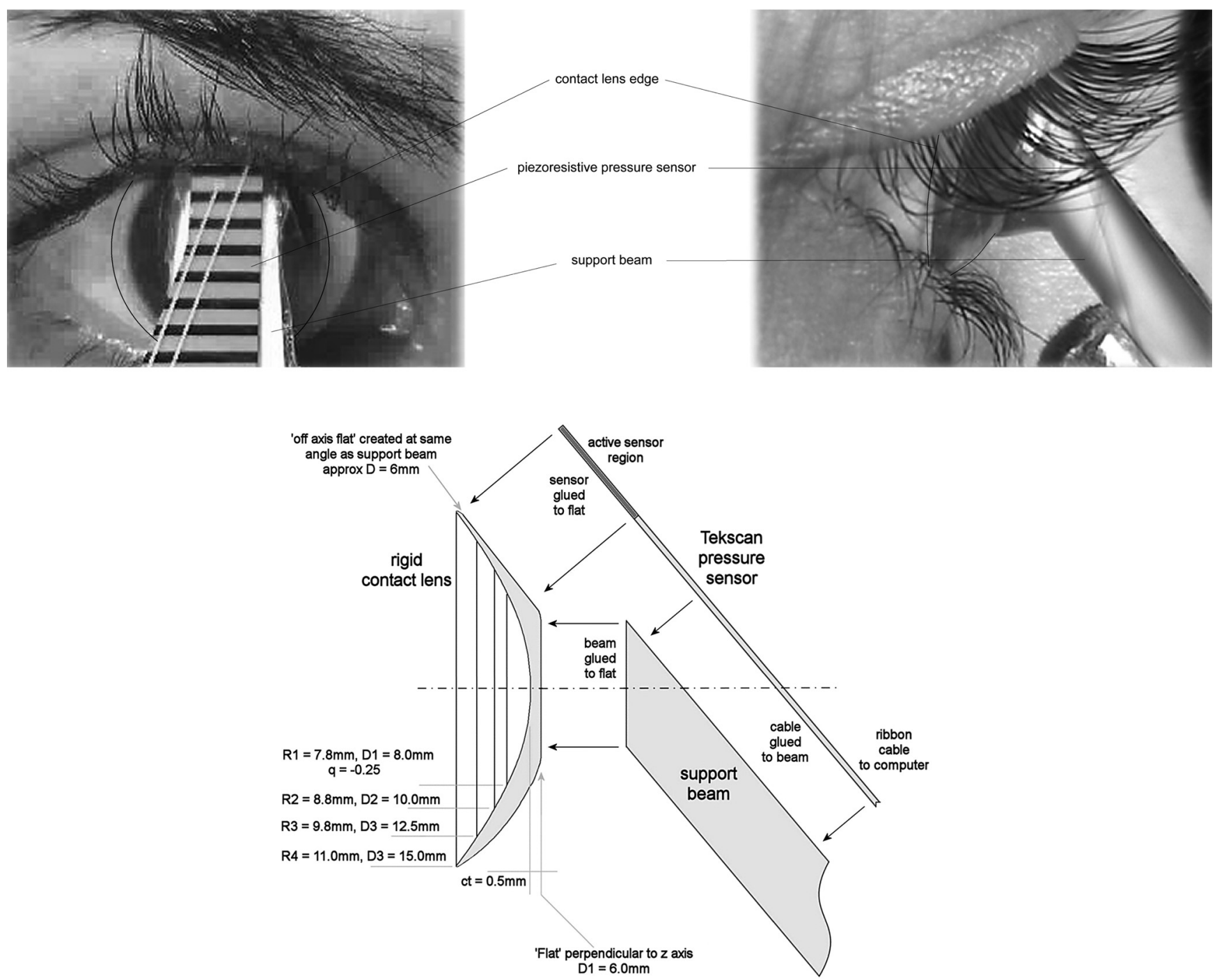

Figure 1. Front view (top left) and side view (top right) of an eyelid pressure measurement using a pressure sensor mounted on a specially designed rigid contact lens with plastic support beam. Schematic of sensor-contact lens combination (lower) with $\mathrm{R}$ (back surface radius of curvature) and D (diameter). All curves are spherical with an asphericity of 0 unless otherwise stated.

\section{Eyelid Pressure Measurement}

The development and validation of the eyelid pressure measurement system has been reported in detail. ${ }^{13}$ The carrier rigid contact lens was designed based on the average corneal topography data of 100 young, healthy subjects, with a back optic zone radius of $7.8 \mathrm{~mm}$ and an eccentricity of $\mathrm{Q}=-0.25 .{ }^{20}$ The contact lens diameter of $15 \mathrm{~mm}$ was chosen so that the upper and the lower eyelids rested on the lens. A specific tactile pressure sensor (Tekscan; Boston, MA) was chosen for this application because it is thin (approximately $0.17 \mathrm{~mm}$ ), has a low pressure limit ( $5 \mathrm{psi}$ ), has good sensitivity, and is suitable for insertion into the eye. The sensor contains piezoresistive ink, which responds to mechanical deformation. The change in electrical resistance is recorded through a cable linked to a computer.

Before use, the pressure sensor attached to a rigid contact lens was conditioned to improve the consistency of its response. The sensor was conditioned with four loads of $25.9 \mathrm{~mm} \mathrm{Hg}$ for 1 minute (with 30-second intervals between loads) less than 60 minutes before use. A calibration procedure was completed to convert the output from the sensor to actual pressure units. Loads of $1,2,2.5,3,3.5,4,5,6,8$, and $10 \mathrm{~mm} \mathrm{Hg}$ were each applied twice, and a linear fit was applied to the average pressure score between 10 and 30 seconds after loading began. The mean coefficient of determination $\left(R^{2}\right)$ ranged from 0.68 to 0.96 , with an average for the 11 subjects of 0.77 .

The sensor-contact lens combination was placed on the eye, with the tears filling between the back surface of the contact lens and cornea (Fig. 1). To obtain a stable measurement of eyelid pressure, the subject was required to maintain constant fixation during measurement, with limited eyelid movement. The most stable measurements were achieved when the non-measurement eye was manually held closed by the subject while fixation in the tested eye was maintained in a direction of approximately $10^{\circ}$ downward gaze. Eyelid pressure measurements (each at $5 \mathrm{~Hz}$ ) were taken until at least two, and preferably three, successful measurements were captured (that is, with the eyelid in the correct position on the sensor for at least 20 seconds). With a mean of 2.8 recordings per subject, each for approximately 12 seconds at $5 \mathrm{~Hz}$, there was a total mean of 168 pressure readings per subject.

Given that the region of the eyelid contacting the sensor could not be visualized during pressure measurement, a front-view video of the eye was recorded to estimate the position of the eyelid margin (Fig. 1). This was exported as .jpg images at $5 \mathrm{~Hz}$ and later analyzed with custom-written image processing software. The eyelid position (i.e., eyelid margin and estimated contact region with the ocular surface) relative to the lower edge of the pressure cell was determined (Fig. 2). 

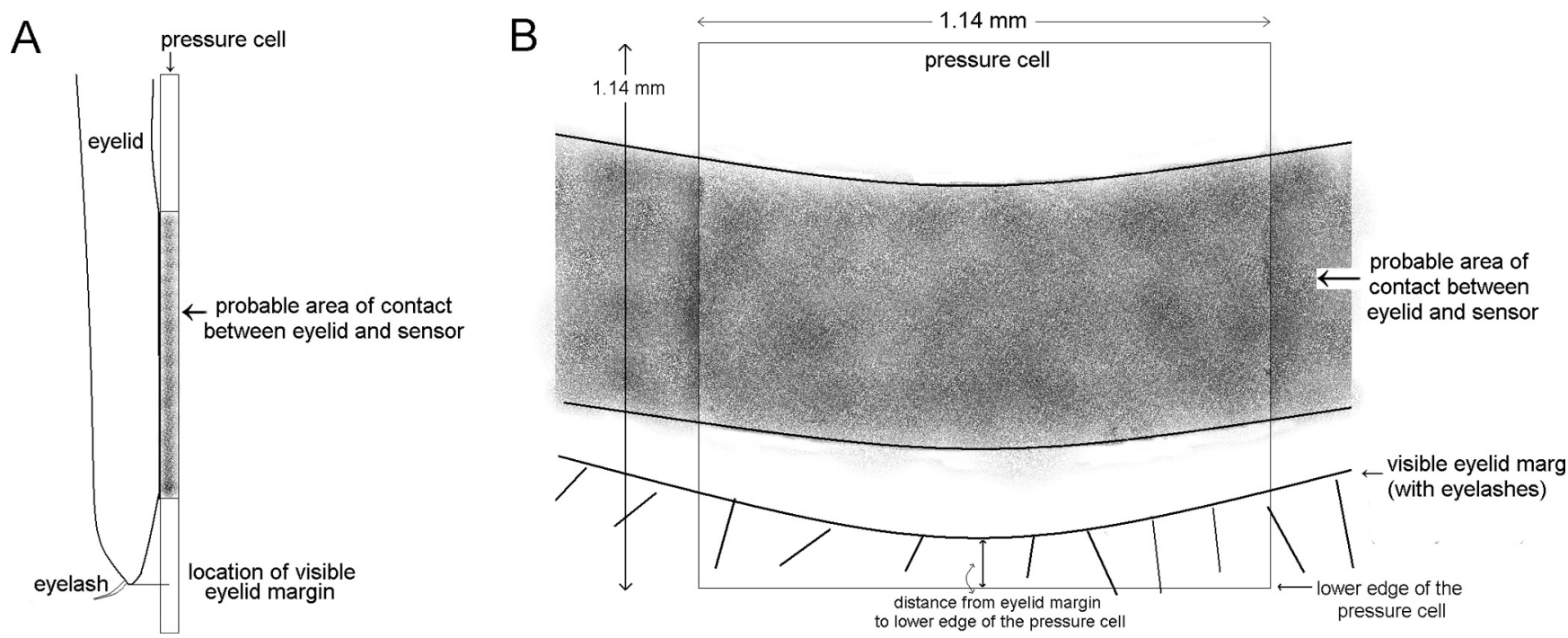

FIGURE 2. Schematic of the eyelid margin contacting the underlying pressure cell. (A) Side view and (B) front view showing the eyelid margin position relative to the lower edge of the pressure cell.

A computer program (written for MATLAB; MathWorks, Natick, MA) was used to filter the sensor's pressure output data dependent on eyelid position with respect to the pressure cell. The first 10 seconds were discarded because of the initial noisy sensor response. ${ }^{13}$ Outliers $(>1.96 \mathrm{SD}$ from the mean) were removed, most often because of eyelid twitches (i.e., small errors of eyelid movement detected in the front video recording). Assuming that the maximum pressure value occurs when the eyelid is in the correct position on the sensor, $50 \%$ of the power of the maximum pressure was used to define a lower boundary limit of data acceptance (upper half criterion). This is equivalent to $1 / \sqrt{ } 2$ of the signal's maximum amplitude or the logarithmic $-3 \mathrm{~dB}$ criterion commonly used in acoustics ${ }^{21}$ and the half-width point spread function used in retinal image metrics. ${ }^{22}$ The raw scores within the upper half criterion were averaged, and the mean raw score was converted to actual pressure units $(\mathrm{mm} \mathrm{Hg}$ ) using the corresponding calibration equation. An example of an eyelid pressure measurement and the corresponding eyelid position is shown in Figure 3. Although the eyelid moved steadily downward over the sensor during the measurement (dashed line), the corresponding pressure measurement was approximately a step function (solid line). Eyelid pressure was zero ( -1 on the graphed normalized scale) after 52 seconds, when the primary contact point of the eyelid moved downward past the pressure cell. Pressure was no longer measured by the sensor (now well under the eyelid), suggesting that a band of the eyelid margin applied pressure to the ocular surface, whereas the remaining eyelid applied very little pressure (below the measurement noise of this technique).

\section{Contact Region between the Eyelids and Cornea}

Contact between the eyelids and the ocular surfaces cannot be directly visualized; however, the work of Kessing ${ }^{1}$ and Korb et al. ${ }^{2}$ suggests that a band of the eyelid margin is in contact with the ocular surface. Three models of contact between the surfaces were investigated. The first model assumed that the eyelid contacts the ocular surface over a width $\geq 1.14 \mathrm{~mm}$ (the width of the sensor's pressure cell; Fig. 4). This is the value calculated using the calibration data, which assumes that the whole pressure cell is loaded during both calibration and measurement. However if the width of primary contact between the eyelid and the cornea is smaller than the pressure cell width $(<1.14 \mathrm{~mm})$, the calibrated pressure must be scaled according to the contact region. We found in preliminary studies that the sensor output represents total force if the cell is only partially loaded. For example, if only half the pressure cell was loaded (contact width, $0.57 \mathrm{~mm}$ ), the pressure reading would be calibrated by a factor of 2 (i.e., 1.14/0.57). The two other models were based on contact widths smaller than the pressure cell width of $1.14 \mathrm{~mm}$, measured by pressure-sensitive paper and Marx's line (Fig. 4). Therefore, three possible models of contact between the eyelid and the ocular surface were investigated along with three corresponding eyelid pressure models-whole cell eyelid pressure, imprint width eyelid pressure, and Marx's line eyelid pressure.

\section{Imprint Width}

To estimate the contact region between the upper eyelid and the ocular surface, pressure-sensitive paper consisting of outer plastic protective layers and inner carbon and adhesive layers (Pressurex; Sensor Products Inc., Madison, NJ) was sourced. This micro paper is used to visualize the pressure distribution between opposing surfaces,

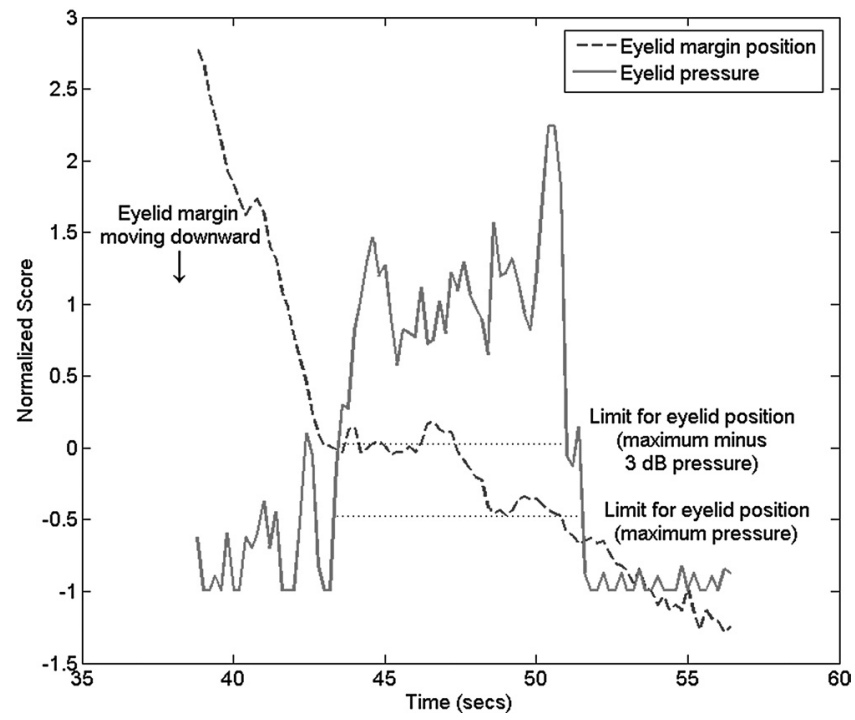

FIGURE 3. Normalized values for eyelid pressure measurement (solid line) and the corresponding eyelid position (relative to the lower edge of the pressure cell, dashed line). Boundaries for eyelid position corresponding to the maximum pressure and upper half criterion are indicated. In this example, the visible eyelid margin was between -0.72 and -0.46 $\mathrm{mm}$ below the edge of the cell during valid pressure readings. The mean raw pressure score (before calibration) corresponding to this eyelid position range was $79.69 \pm 28.46$ based on 32 data points. 


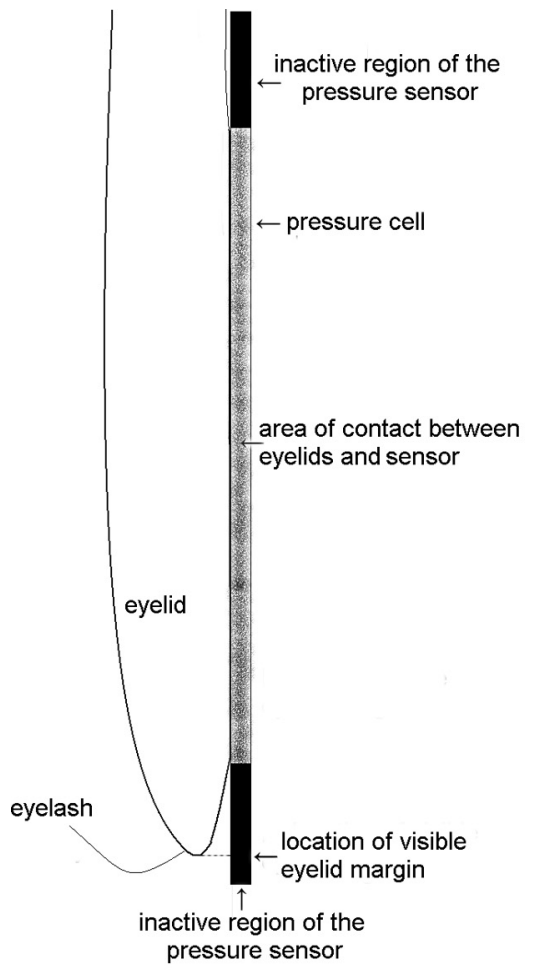

A) "Whole cell" eyelid pressure (contact width $>1.14 \mathrm{~mm}$ )

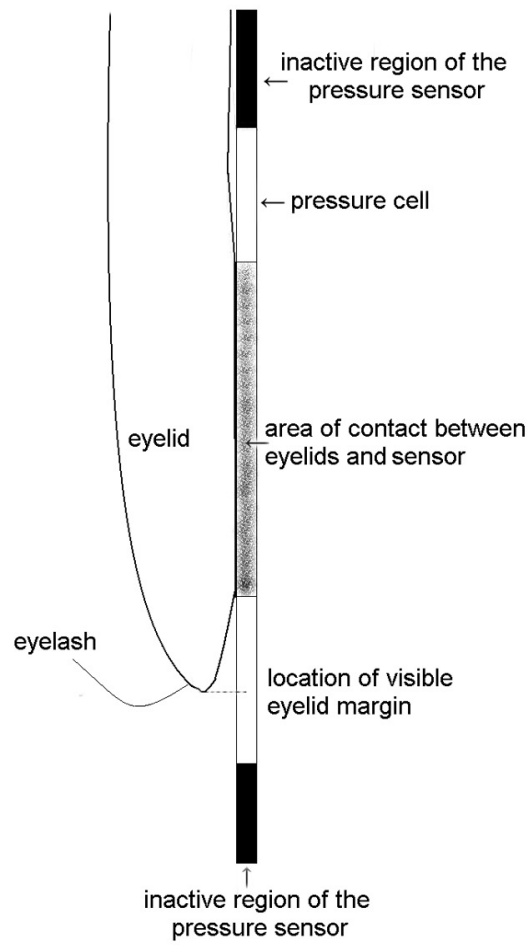

B) "Imprint width" eyelid pressure (mean contact width $=0.60 \mathrm{~mm}$ )

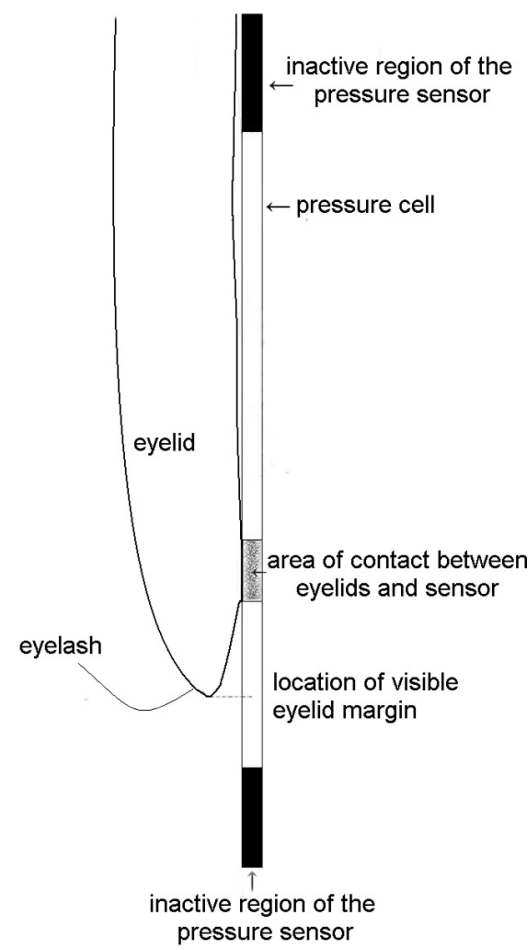

C) "Marx's line" eyelid pressure (mean contact width $=0.09 \mathrm{~mm}$ )

Figure 4. Possible models of eyelid contact with the pressure cell. (A) The eyelid contacts the whole cell. (B) Contact is over the imprint width determined by micro paper imprint. (C) Contact over Marx's line width determined by lissamine-green staining and digital photography.

such as during lamination or between industrial rollers. When pressure is applied, a carbon imprint is produced on the adhesive layer.

The specifications suggest a minimum pressure sensitivity of $2 \mathrm{psi}$ or $103.4 \mathrm{~mm} \mathrm{Hg}$; however, lower pressures were recorded in trials with known pressures (as low as $4.6 \mathrm{~mm} \mathrm{Hg}$ ) when the applanation surface was wet. This explains why eyelid margin pressure could be measured despite pressure that was lower than the minimum quoted pressure sensitivity of the paper.

Segments of micro paper (Pressurex; Sensor Products Inc.) were adhered to the flat area of the custom contact lenses with double-sided adhesive tape. The subject's eye was anesthetized ( $0.4 \%$ benoxinate), and the contact lens with attached paper was placed on the eye while the eyelids were held open. The upper eyelid was then released to contact the pressure-sensitive paper for approximately 10 seconds, during which time the subject was instructed to try to refrain from blinking. The eyelid was then lifted away from the paper, and the contact lens was removed from the eye. If the subject blinked, the test was repeated.

Five separate contact imprints were collected for each subject, and digital images of the imprints were obtained for analysis (Fig. 5). Using custom software, points were chosen along both the upper and the lower edges of the imprint, each fit with a fourth-order polynomial. The mean distance between the upper and lower boundaries (imprint width) was calculated and averaged for each subject (Fig. 6).

\section{Marx's Line}

Lissamine green was applied to the superior and inferior bulbar conjunctiva of the right eye for each subject using an impregnated strip
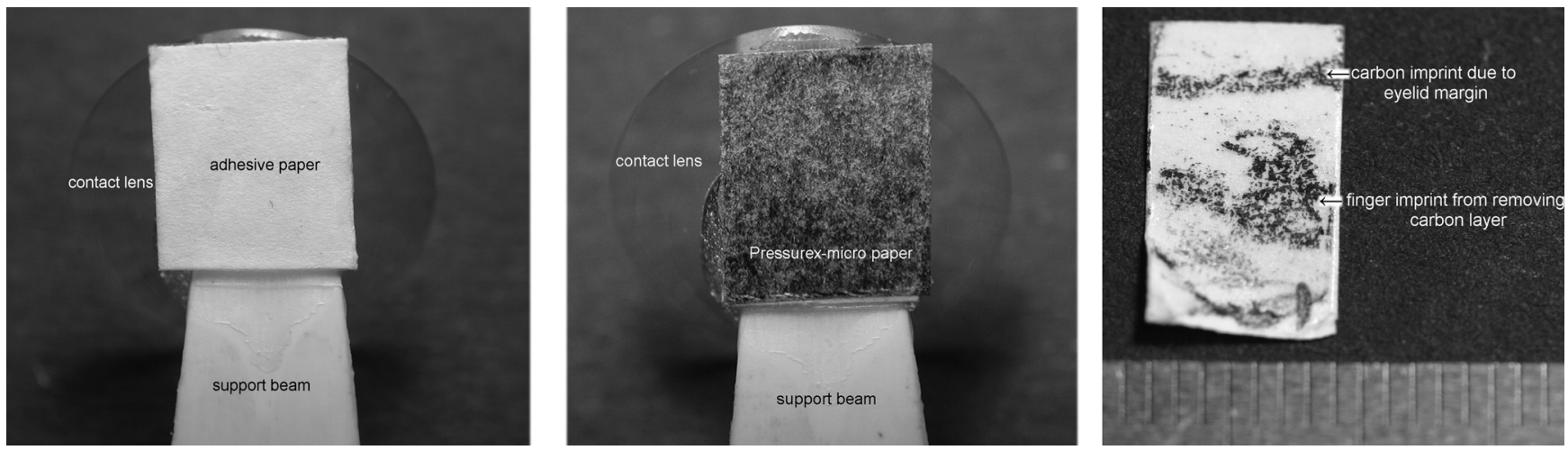

FiguRE 5. Example of pressure-sensitive micro paper. Adhesive layer attached to the contact lens (left), carbon layer placed in contact with the adhesive layer (middle), and carbon imprint on the adhesive layer after exposure to eyelid margin pressure and removal of the carbon layer (right). 
Pressure sensitive paper imprint

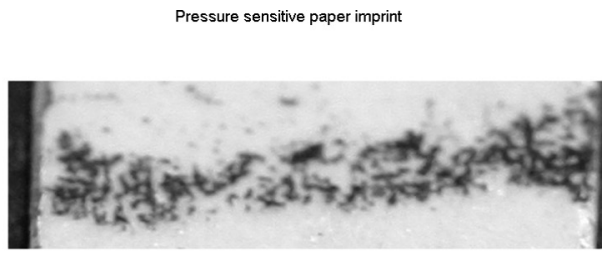

Digital image
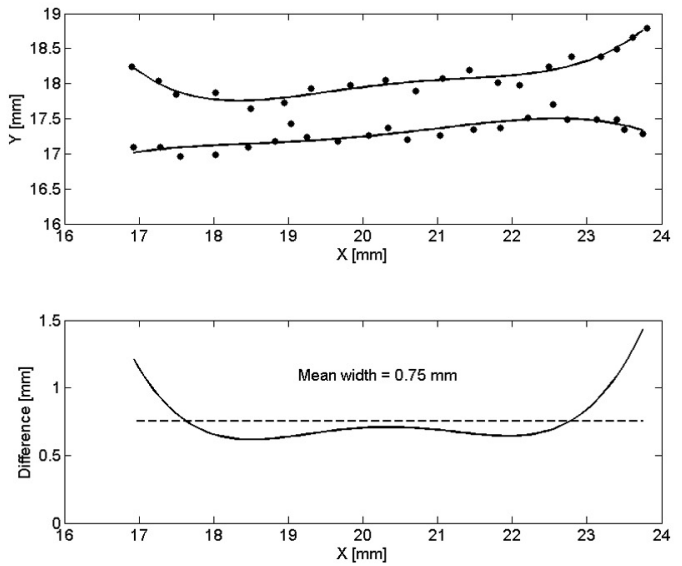

Lissamine-green stained Marx's line
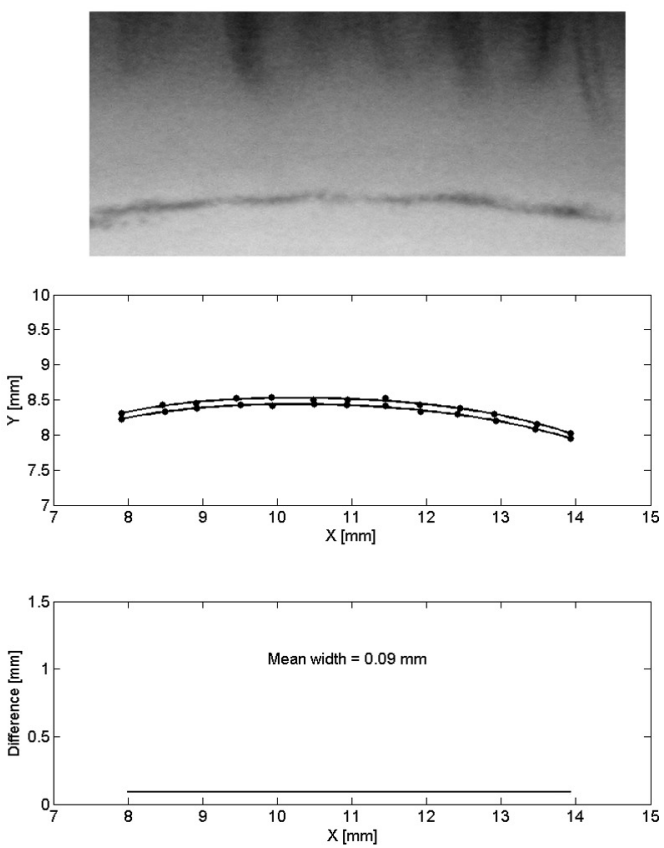

Figure 6. Examples of the digital images (top) and analysis (middle and lower) of a contact imprint width (mean, $0.75 \mathrm{~mm}$ ) and a Marx's line width (mean, $0.09 \mathrm{~mm}$ ). Both widths were averaged over approximately the central $6 \mathrm{~mm}$ of the eyelids.

wetted with two drops of sterile saline, and the excess fluid was allowed to run off. Digital images of the upper eyelid Marx's line were captured by everting the upper eyelid. These images were analyzed with custom software, similar to that used to analyze the contact imprints, with points chosen along the upper and lower boundaries over a 6-mm central portion of the eyelid. This length was chosen to ensure that Marx's line would be in focus in the digital image for each subject. The upper and lower boundaries were fit with fourth-order polynomials, and the average distance between the boundaries (mean Marx's line width) was calculated for each subject (Fig. 6).

\section{Results}

Eyelid pressure was calculated based on the three models of contact between the sensor and eyelid: whole cell, imprint width, and Marx's line (Fig. 5). These pressure estimates, along with the raw score values for each measurement, can be seen in Table 1 . The mean whole cell eyelid pressure was $3.8 \pm 0.7$ $\mathrm{mm} \mathrm{Hg}$ (range, $2.8-5.1 \mathrm{~mm} \mathrm{Hg}$ ) for the 11 subjects (Table 1 ).
The micro paper (Pressurex; Sensor Products Inc.) imprints showed a defined band of pressure between the eyelid margin and the ocular surface. The mean width of the contact imprints was $0.60 \pm 0.16 \mathrm{~mm}$ (range, $0.33-0.84 \mathrm{~mm}$ ). When eyelid pressure was scaled for each subject using the contact imprint width, the mean imprint width eyelid pressure for the 11 subjects was $8.0 \pm 3.4 \mathrm{~mm} \mathrm{Hg}$ (range, 4.4-14.4 mm Hg) (Table 1).

The mean Marx's line width was $0.09 \pm 0.02 \mathrm{~mm}$. The mean eyelid pressure adjusted for a contact width of Marx's line (Marx's line eyelid pressure) was $55 \pm 26 \mathrm{~mm} \mathrm{Hg}$ (range, 32-115 mm Hg) (Table 1).

\section{DisCuSSION}

The contact imprint widths measured in this study with pressure-sensitive paper provide a new estimate of contact width between the upper eyelid and the ocular surface. The imprints confirm previous suggestions that the eyelid margin is the primary contact region with the ocular surface and may rep-

Table 1. Eyelid Pressure Using the Three Models: Whole Cell, Imprint Width, and Marx's Line

\begin{tabular}{|c|c|c|c|c|c|c|}
\hline Subject & $\begin{array}{c}\text { Raw } \\
\text { Scores }\end{array}$ & $\begin{array}{c}\text { Whole Cell } \\
\text { Eyelid Pressure } \\
\text { (mm Hg) }\end{array}$ & $\begin{array}{c}\text { Imprint Width } \\
(\mathrm{mm})\end{array}$ & $\begin{array}{l}\text { Imprint Width } \\
\text { Eyelid Pressure } \\
\text { (mm Hg) }\end{array}$ & $\begin{array}{c}\text { Marx's Line Width } \\
\text { (mm) }\end{array}$ & $\begin{array}{c}\text { Marx's Line } \\
\text { Eyelid Pressure } \\
\text { (mm Hg) }\end{array}$ \\
\hline 1 & $41,63,40$ & $3.2 \pm 0.6$ & $0.55 \pm 0.20$ & $6.6 \pm 2.6$ & $0.07 \pm<0.01$ & $50 \pm 8$ \\
\hline 2 & $81,119,91$ & $5.1 \pm 0.6$ & $0.40 \pm 0.05$ & $14.4 \pm 2.4$ & $0.08 \pm 0.02$ & $77 \pm 19$ \\
\hline 3 & 41,67 & $2.9 \pm 0.6$ & $0.74 \pm 0.08$ & $4.5 \pm 1.0$ & $0.09 \pm<0.01$ & $38 \pm 8$ \\
\hline 4 & $77,58,85$ & $4.9 \pm 0.6$ & $0.62 \pm 0.05$ & $9.0 \pm 1.2$ & $0.05 \pm 0.01$ & $115 \pm 25$ \\
\hline 5 & $35,47,51$ & $3.4 \pm 0.3$ & $0.39 \pm 0.05$ & $9.9 \pm 1.5$ & $0.12 \pm 0.01$ & $32 \pm 5$ \\
\hline 6 & $70,83,38$ & $3.9 \pm 0.7$ & $0.62 \pm 0.13$ & $7.0 \pm 2.0$ & $0.09 \pm<0.01$ & $46 \pm 9$ \\
\hline 7 & $61,41,49$ & $4.1 \pm 0.4$ & $0.65 \pm 0.25$ & $7.2 \pm 2.8$ & $0.08 \pm 0.01$ & $56 \pm 6$ \\
\hline 8 & $46,47,25$ & $4.0 \pm 0.8$ & $0.33 \pm 0.04$ & $13.7 \pm 3.2$ & $0.09 \pm 0.02$ & $50 \pm 15$ \\
\hline 9 & 23,34 & $2.8 \pm 0.3$ & $0.73 \pm 0.01$ & $4.4 \pm 0.5$ & $0.10 \pm 0.01$ & $33 \pm 5$ \\
\hline 10 & $36,37,37$ & $3.5 \pm<0.1$ & $0.84 \pm 0.23$ & $4.8 \pm 1.3$ & $0.12 \pm 0.02$ & $34 \pm 5$ \\
\hline 11 & $63,64,38$ & $4.4 \pm 0.8$ & $0.72 \pm 0.10$ & $7.0 \pm 1.7$ & $0.06 \pm 0.01$ & $79 \pm 19$ \\
\hline Mean \pm SD & - & $3.8 \pm 0.7$ & $0.60 \pm 0.16$ & $8.0 \pm 3.4$ & $0.09 \pm 0.02$ & $55 \pm 26$ \\
\hline
\end{tabular}

Raw scores are the values obtained from the pressure sensor before calibration. 
resent the zone now defined as the eyelid wiper. ${ }^{2,3}$ An average contact width of $0.60 \mathrm{~mm}$ (range, $0.33-0.84 \mathrm{~mm}$ ) discounts the whole cell eyelid pressure model, which assumes a contact width greater than the pressure cell width of $1.14 \mathrm{~mm}$. Primary contact by a band of the eyelid margin can also be seen from the data in Figure 3. When the eyelid margin moved downward past the pressure cell, the eyelid no longer exerted significant pressure on the underlying surface.

Marx's line is an anatomic feature present in nearly everyone; the mean width reported in this study was $0.09 \mathrm{~mm}$, in agreement with previous measurements., ${ }^{93}$ Due to its anatomic structure and staining properties, it is thought to have frictional contact with the ocular surfaces and can be suggested to be the narrowest possible contact region. The static upper eyelid pressure calculated, assuming that only Marx's line contacts the ocular surface, resulted in an average pressure of $55 \mathrm{~mm} \mathrm{Hg}$ (range, 32-115 mm Hg). This pressure was high considering that intraocular pressure is usually approximately $15 \mathrm{~mm} \mathrm{Hg}$ and that peak pressure used to deform the cornea by noncontact tonometry is approximately $90 \mathrm{~mm} \mathrm{Hg}{ }^{24}$ Therefore, it was unlikely that the upper eyelid contacted the ocular surface over a width as narrow as Marx's line.

The imprint width eyelid pressure model based on carbon imprints of the contact between the upper eyelid and ocular surface, in our opinion, provides the most reliable estimate of static upper eyelid pressure. Imprints in the corneal surface due to the upper eyelid have been measured after steady fixation tasks. ${ }^{23}$ The mean widths of the peak-to-peak depressions caused by the upper eyelid were $1.3 \mathrm{~mm}$ and $1.4 \mathrm{~mm}$ after steady fixation conditions at $20^{\circ}$ and $40^{\circ}$ downward gaze, respectively. ${ }^{23}$ It can be assumed that to create the peak-valleypeak profile, tissue or fluid would be distributed away from the depression. These findings are consistent with the mean imprint contact width $(0.60 \mathrm{~mm})$ in this study smaller than the corneal depression peak-to-peak widths $(1.3$ and $1.4 \mathrm{~mm})$ previously measured.

Further confirmation of the validity of these measurements is the calculation of eyelid pressure from eyelid tension. Although tension does not necessarily directly relate to pressure, the calculation gives some insight into whether the eyelid pressure measurements seem reasonable. The eyelid force estimated from measurements with an eyelid tensiometer ${ }^{25}$ is approximately $30 \mathrm{mN} .^{26}$ In comparison, the mean eyelid pressure of $8 \mathrm{~mm} \mathrm{Hg}$ measured by this study with a piezoresistive pressure sensor is equivalent to an eyelid force of $19 \mathrm{mN}$, showing reasonable agreement.

Some comparison can be made to previous eyelid pressure measurements, though this comparison is limited by different techniques and measurement conditions. Miller ${ }^{12}$ used a manometer system to measure the pressure of a number of subjective blink conditions: light, gentle, deliberate, and hard squeeze. The one most comparable to static eyelid pressure was the light blink condition. Its mean result was $2.8 \pm 2.2 \mathrm{~mm}$ $\mathrm{Hg}$ eyelid pressure. Lydon and Tait ${ }^{11}$ appeared to have an improved technique, but did not provide quantitative values of eyelid pressure. Shikura et al. (IOVS 1993;34:ARVO Abstract 1250) found that the average eyelid pressure during normal lid closure was $1.7 \mathrm{~mm} \mathrm{Hg}$ (range, -0.50 to $6.7 \mathrm{~mm} \mathrm{Hg}$ ). These pressures are lower than the mean measured by all three models in this study, most likely because of the nature of the manometer systems, which would not measure the localized pressure of the eyelid margin but rather would determine average pressure from the upper eyelid over a much larger area than our technique measured.

In this study, the imprint width eyelid pressure varied from 4.4 to $14.4 \mathrm{~mm} \mathrm{Hg}$. Given that this range was larger than originally expected, pressure and imprint measurements were repeated on two subjects with high and low results to confirm repeatability. The initial pressure measurement for the first subject was $4.5 \pm 1.0 \mathrm{~mm} \mathrm{Hg}$, and the repeated measurement was $4.0 \pm 1.5 \mathrm{~mm} \mathrm{Hg}$; the initial pressure measurement for the second subject was $14.4 \pm 2.4 \mathrm{~mm} \mathrm{Hg}$, and the repeated measurement was $13.5 \pm 3.4 \mathrm{~mm} \mathrm{Hg}$. With comparable repeated measurements for these subjects, it seems possible that there may be a large range of eyelid pressure between persons.

It should be acknowledged that there is some thickness of the contact lens and pressure-sensitive paper (approximately $0.7 \mathrm{~mm}$ ) between the eyelid and cornea during the measurement that may slightly alter the contact relationship between the surfaces. However, the contact imprint technique directly relates to the eyelid pressure measurement because both are taken using the same thickness of the underlying contact lens. Pilot investigations found that the eyelid had to be in contact with the paper for a minimum of 10 seconds for an imprint to be recorded. During this time there might have been some small movement of the eyelid, but it is not known how long the eyelid has to stay in a fixed position to cause an imprint. It can be concluded that the true area of contact between the eyelid margin and the surface of the paper may be slightly smaller, but probably not larger, than the measured group mean value of $0.60 \mathrm{~mm}$

The pressure of the tear film meniscus at the eyelid margin should also be considered in relation to these results. Based on a surface tension of $0.0454 \mathrm{~N} / \mathrm{m}^{27}$ and an average tear meniscus radius of curvature of $0.365 \mathrm{~mm},{ }^{28}$ the pressure of the meniscus is $125 \mathrm{~N} / \mathrm{m}^{2}$ or $0.9 \mathrm{~mm} \mathrm{Hg} .{ }^{26}$ This force acts in a direction away from the cornea (opposite to eyelid pressure) and may be involved in the wavelike corneal surface change (peaks and valley) previously recorded. ${ }^{23}$ However, it is unlikely to have an impact on eyelid pressure measurements because eyelid pressure and tear meniscus pressure have different locations on the sensor and are unlikely to be located within the same pressure cell (1.14-mm width), even if the sensor was able to register negative outward pressure. Therefore, it is unlikely that tear meniscus pressure influenced eyelid pressure measurements in this study.

It should be noted that measurements in this study were for a static eyelid and that eyelid pressure may change during blinking. Previous studies examining a number of blinking conditions found that eyelid pressure increased by a factor of 18 between light blinks and hard squeezes of the eyelids. ${ }^{12}$ Further experimentation with piezoresistive sensors may lead to an understanding of the dynamic nature of eyelid pressure during blinking.

In summary, using imprint width as the best estimate of contact between the eyelid and the pressure sensor, the mean eyelid pressure of a group of young adult subjects was $8.0 \pm$ $3.4 \mathrm{~mm} \mathrm{Hg}$. Contact imprints confirm previous suggestions that a band of the eyelid margin is the primary contact with the ocular surface (mean width, $0.60 \pm 0.16 \mathrm{~mm}$ ).

\section{Acknowledgments}

The authors thank D. Robert Iskander for his assistance with software for aspects of the analysis.

\section{References}

1. Kessing SV. A new division of the conjunctiva on the basis of x-ray examination. Acta Opbthalmol. 1967;45(5):680-683.

2. Korb DR, Greiner JV, Herman JP, et al. Lid-wiper epitheliopathy and dry-eye symptoms in contact lens wearers. CLAO J. 2002; 28(4):211-216.

3. Korb DR, Herman JP, Greiner JV, et al. Lid wiper epitheliopathy and dry eye symptoms. Eye Contact Lens. 2005;31(1):2-8.

4. Pult H, Purslow C, Berry M, Murphy PJ. Clinical tests for successful contact lens wear: relationship and predictive potential. Optom Vis Sci. 2008;85(10):E924-E929. 
5. Varikooty J, Srinivasan S, Jones L. Atypical manifestation of upper lid margin staining in silicone hydrogel lens wearers with symptoms of dry eye. Contact Lens Anterior Eye. 2008;31:44-46.

6. Marx E. Uber anatomie, physiologie und pathologie des augenlidrandes un der tranenpunkte. Graefes Arch Opbthalmol. 1924; 114:465-482.

7. Hughes C, Hamilton L, Doughty MJ. A quantitative assessment of the location and width of Marx's line along the marginal zone of the human eyelid. Optom Vis Sci. 2003;80(8):564-572.

8. Norn M. Meibomian orifices and Marx's line: studied by triple vital staining. Acta Opbthalmol. 1985;63(6):698-700.

9. Doughty MJ, Naase T, Donald C, Hamilton L, Button NF. Visualisation of Marx's line along the marginal eyelid conjunctiva of human subjects with lissamine green dye. Opbthal Physiol Opt. 2004; 24(1):1-7.

10. Parsons J. The Patbology of the Eye. Vol. 1. London: Hodder and Stoughton; 1904.

11. Lydon D, Tait A. Lid pressure: its measurement and probable effects on the shape and form of the cornea-rigid contact lens system. J Br Contact Lens Assn. 1988;11(1):11-22.

12. Miller D. Pressure of the lid on the eye. Arch Ophthalmol. 1967; 78(3):328-330

13. Shaw AJ, Davis BA, Collins MJ, Carney LG. A technique to measure eyelid pressure using piezoresistive sensors. IEEE Trans Biomed Eng. 2009;56(10):2512-2517.

14. Mengher LS, Pandher KS, Bron AJ. Non-invasive tear film break-up time: sensitivity and specificity. Acta Opbthalmol. 1986;64(4): $441-444$.

15. Jupiter D, Karesh J. Ptosis associated with PMMA/rigid gas permeable contact lens wear. CLAO J. 1999;25(3):159-162.

16. Kersten RC, de Conciliis C, Kulwin DR. Acquired ptosis in the young and middle-aged adult population. Opbthalmology. 1995; 102(6): $924-928$.
17. Thean JH, McNab AA. Blepharoptosis in RGP and PMMA hard contact lens wearers. Clin Exp Optom. 2004;87(1):11-14.

18. van den Bosch WA, Leenders I, Mulder P. Topographic anatomy of the eyelids, and the effects of sex and age. Br J Ophthalmol. 1999;83(3):347-352.

19. Nemoto Y, Morikawa K, Kaneko H. A case of blepharoptosis associated with long-term use of soft contact lenses. Nippon Ganka Gakkai Zassbi. 2008;112(10):876-881.

20. Read SA, Collins MJ, Carney LG, Franklin RJ. The topography of the central and peripheral cornea. Invest Ophthalmol Vis Sci. 2006; 47(4):1404-1415.

21. Harrington $\mathrm{J}$, Cassidy $\mathrm{S}$. Techniques in speech acoustics. In: Ide $\mathbf{N}$, Véronis J, eds. Text, Speech and Language Technology Series. Volume 8. New York: Springer; 1999.

22. Thibos LN, Hong X, Bradley A, Applegate RA. Accuracy and precision of objective refraction from wavefront aberrations. $J$ Vis. 2004;4(4):329-351.

23. Shaw AJ, Collins MJ, Davis BA, Carney LG. Eyelid pressure: inferences from corneal topographic changes. Cornea. 2009;28(2): 181- 188 .

24. Kaneko M, Tokuda K, Kawahara T. Dynamic sensing of human eye. Proc IEEE Int Conference Robotics Automation. 2005;28712876.

25. Ehrmann K, Francis I, Stapleton F. A novel instrument to quantify the tension of upper and lower eyelids. Contact Lens Anterior Eye. 2001;24:65-72.

26. Jones MB, Fulford GR, Please CP, McElwain DLS, Collins MJ. Elastohydrodynamics of the eyelid wiper. Bull Math Biol. 2008; 70(2):323-343

27. Nagyova B, Tiffany JM. Components responsible for the surface tension of human tears. Curr Eye Res. 1999;19(1):4-11.

28. Yokoi N, Bron A, Tiffany J, Brown N, Hsuan J, Fowler C. Reflective meniscometry: a non-invasive method to measure tear meniscus curvature. Br J Opbthalmol. 1999;83(1):92-97. 